\title{
COVID-19 pandemic in BRICS countries and its association with socio-economic and demographic characteristics, health vulnerability, resources, and policy response
}

\author{
Jingmin Zhu', Wenxin Yan², Lin zhu ${ }^{3}$ and Jue Liü 2,4,5*
}

\begin{abstract}
Background: Little attention has been paid to the comparison of COVID-19 pandemic responses and related factors in BRICS (Brazil, Russia, India, China, and South Africa) countries. We aimed at evaluating the association of daily new COVID-19 cases with socio-economic and demographic factors, health vulnerability, resources, and policy response in BRICS countries.
\end{abstract}

Methods: We conducted a cross-sectional study using data on the COVID-19 pandemic and other indicators of BRICS countries from February 26, 2020 to April 30, 2021. We compared COVID-19 epidemic in BRICS countries and analyzed related factors by log-linear Generalized Additive Model (GAM) models.

Results: In BRICS countries, India had the highest totally of confirmed cases with 18.76 million, followed by Brazil (14.45 million), Russia (4.81 million), and South Africa (1.58 million), while China (0.10 million) had the lowest figure. South Africa had the lowest rate of administered vaccine doses (0.18 million) among BRICS countries as of April 30, 2021. In the GAM model, a 1 unit increase in population density and policy stringency index was associated with a $5.17 \%$ and $1.95 \%$ growth in daily new COVID-19 cases $(P<0.001)$, respectively. Exposure-response curves for the effects of policy stringency index on daily new cases showed that there was a rapid surge in number of daily new COVID-19 cases when the index ranged from 0 to 45. The number of infections climbed slowly when the index ranged from 46 to 80 , and decreased when the index was above $80(P<0.001)$. In addition, daily new COVID-19 cases (all $P<0.001$ ) were also correlated with life expectancy at birth $(-1.61 \%)$, extreme poverty $(8.95 \%)$, human development index $(-0.05 \%)$, GDP per capita $(-0.18 \%)$, diabetes prevalence $(0.66 \%)$, proportion of population aged 60 and above $(2.23 \%)$, hospital beds per thousand people $(-0.08 \%)$, proportion of people with access to improved drinking water (-7.40\%), prevalence of open defecation (0.69\%), and annual tourist/visitor arrivals (0.003\%), after controlling other confounders. Different lag structures showed similar results in the sensitivity analysis.

Conclusions: Strong policy response is crucial to control the pandemic, such as effective containment and case management. Our findings also highlighted the importance of reducing socio-economic inequalities and strengthening the resilience of health systems to better respond to public health emergencies globally.

\footnotetext{
*Correspondence: jueliu@bjmu.edu.cn

2 Department of Epidemiology and Biostatistics, School of Public Health,

Peking University, Haidian District, No. 38, Xueyuan Road, Beijing 100191,

China

Full list of author information is available at the end of the article
}

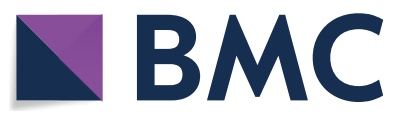

(c) The Author(s) 2021. Open Access This article is licensed under a Creative Commons Attribution 4.0 International License, which permits use, sharing, adaptation, distribution and reproduction in any medium or format, as long as you give appropriate credit to the original author(s) and the source, provide a link to the Creative Commons licence, and indicate if changes were made. The images or other third party material in this article are included in the article's Creative Commons licence, unless indicated otherwise in a credit line to the material. If material is not included in the article's Creative Commons licence and your intended use is not permitted by statutory regulation or exceeds the permitted use, you will need to obtain permission directly from the copyright holder. To view a copy of this licence, visit http://creativecommons.org/licenses/by/4.0/. The Creative Commons Public Domain Dedication waiver (http://creativeco mmons.org/publicdomain/zero/1.0/) applies to the data made available in this article, unless otherwise stated in a credit line to the data. 
Keywords: COVID-19, BRICS countries, Policy response, Associated factors

\section{Background}

Coronavirus disease 2019 (COVID-19) has become a global public health issue. Figures from the World Health Organization (WHO) showed that more than 150.99 million COVID-19 cases have been confirmed worldwide, with over 3.17 million deaths as of May 1, 2021 [1]. Comprehensive measures have been taken across the world to curb the spread of the new severe acute respiratory syndrome coronavirus (SARS-CoV-2). The COVID-19 pandemic has affected countries in different ways [2]. BRICS constitute a political and economic grouping of countries (Brazil, Russia, India, China, and South Africa) undergoing rapid economic development, making up almost half of the world's population. As of April 30, the number of SARS-CoV-2 infections in BRICS countries has reached 39.77 million, accounting for more than a quarter (26.3\%) of the global total.

Previous analyses have focused on experiences and challenges in a specific country or globally [3-9]. Rocha et al. [6] found that existing socioeconomic inequalities rather than other risk factors have affected the course of COVID-19 epidemic in Brazil. Jefferies et al. [10] reported that New Zealand's strong response to COVID19 resulted in low relative burden of disease, low levels of population disease disparities from Feb 2 to May 13, 2020. Romaniuk et al. compared health system outcomes in the BRICS countries during 2000-2017 and found a weak correlation with economic background [11]. Studies are needed to determine how exactly socio-economic characteristics, resources, and policy affect health system outcomes [11].

The influence of BRICS countries in the international arena has risen enormously in recent decades [12]. However, little attention has been paid to the comparison of COVID-19 pandemic responses and related factors in BRICS countries, despite their increasing global significance as individual countries and as an economic grouping [13]. Comparing COVID-19 situations and exploring its related factors in BRICS countries are important to better understand the spread of SARS-CoV-2 and pandemic control. This work is aimed at evaluating the association of daily new COVID-19 cases with socioeconomic and demographic factors, health vulnerability, resources, and policy response among BRICS countries through a comparative study.

\section{Methods \\ Data collection}

This study analyzed data on the COVID-19 pandemic and other indicators of BRICS countries (Brazil, Russia, India, China, and South Africa) as of April 30, 2021. The indicators were selected based on availability and authenticity of data, as well as the assumption that they are the best available direct indicators related to the outcomes $[6,14,15]$. All data used in this study were collected from the public databases of Our World in Data, WHO, United Nations, World Bank, and other references.

\section{Data on response and COVID-19 outcomes}

We used daily COVID-19 data from Our World in Data (https://ourworldindata.org/coronavirus-source-data) [16], which collected different sets of data related to COVID-19 from multiple sources globally. The number of COVID-19 cases in the Our World in Data database was sourced from Johns Hopkins University dashboard, which has been publishing updates on confirmed cases and deaths for all countries since January 22, 2020 [17]. The total administered vaccine doses, total confirmed COVID-19 cases, and transmission classification as of April 30, 2021 were derived from WHO (https://covid19. who.int/). In terms of transmission classification, WHO has defined four transmission scenarios for COVID-19, including no cases (countries with no cases), sporadic cases (countries with one or more cases, imported or locally detected), clusters of cases (countries experiencing cases, clustered in time, geographic location, and/ or by common exposure), and community transmission (countries experiencing larger outbreaks of local transmission) [1].

In previous studies, a daily policy stringency index was used to reflect the level of policy response [6, 18-20]. The national stringency index, compiled from a global database of pandemic policies (Oxford COVID-19 Government Response Tracker, OxCGRT), addresses the need for continuously updated, readily usable and comparable information on policy measures [18]. This database enables policymakers and researchers to explore the empirical effects of policy responses on the spread of COVID-19. The policy stringency index ranged from a score of 0 to 100 . The higher the score, the higher the level of policy response. 
Data on socio-economic and demographic characteristics, health vulnerability and resources

The data on socio-economic and demographic characteristics (including total population, population density, life expectancy at birth, extreme poverty, human development index and GDP per capita), health vulnerability (including diabetes prevalence, cardiovascular death rate and proportion of population aged 60 and above) as well as health system resources (hospital beds per thousand people) of BRICS countries were sourced from the public database of the United Nations (UN) (http://data.un. org/) and World Bank (https://data.worldbank.org/). The UN's public tourism and transport database was also used to compile tourist/visitor arrivals to reflect population mobility.

Given that water and sanitation resources might also affect the spread of COVID-19, we included the proportion of people with access to improved drinking water and prevalence of open defecation as potential indicators $[6,21]$. The proportion of people with access to improved drinking water was derived from previous studies in BRICS countries [21]. As one of the indicators of the sustainable development goals, the prevalence of open defecation was obtained from the latest UN report (https:// www.sdg6data.org/indicator/6.2.1a).

\section{Statistical analysis}

We used descriptive statistics and estimated Pearson coefficients for bilateral correlations between socioeconomic and demographic factors, health vulnerability, resources, policy response to the COVID-19 pandemic and daily new cases in BRICS countries.

A log-linear Generalized Additive Model (GAM) model was used to analyze the associations between the expectation of the response variable (daily new cases) and the nonparametric predictor variables (such as policy stringency index) in this study. The GAM model is a combination of the generalized linear model and the additive model, which has been widely used in the research of infectious diseases using a connection function to establish the relationship between response variable and predictor variables [22-24]. The formula of the model is defined as follows:

$$
\begin{aligned}
\log Y_{t}= & \alpha+\beta_{1} \operatorname{Var}_{1 t}+\beta_{2} \operatorname{Var}_{2 t}+\beta_{2} \operatorname{Var}_{3 t}+\ldots+\beta_{n} \operatorname{Var}_{n t} \\
& ++s(P S I, d f)+\text { Country }_{i}+\text { Week }+ \text { day }_{t}
\end{aligned}
$$

In this formula, $\log \left(Y_{t}\right)$ is the $\log$-transformed of the number of daily new COVID-19 cases on day $\mathrm{t}_{\mathrm{t}} \alpha$ is the intercept; $d f$ is the degree of freedom; $V a r_{1 t} \sim V a r_{n t}$ represent the variables of socio-economic and demographic factors (population density, life expectancy at birth, extreme poverty, human development index, GDP per capita), health vulnerability (diabetes prevalence, and proportion of population aged 60 and above), health system, water and sanitation resources (hospital beds per thousand people, proportion of people with access to improved drinking water, prevalence of open defecation), and population mobility (tourist/visitor arrivals). $P S I$ is the policy response (policy stringency index) to the COVID-19 pandemic on day ${ }_{\mathrm{t}} s()$ refers to a thin plate spline function based on the penalized smoothing spline to fit the long-term trend of the time. Country ${ }_{i}$ is a categorical variable for BRICS countries. Week is a categorical variable indicating the date of the week to control the day of the week effect, because the number of daily new cases might always be higher on one day of the week (such as Monday) as reported in previous studies [23], while $d a y_{t}$ is the number of days with COVID-19 cases in a country to capture the day fixed effect. The econometric problems (normality, multicollinearity, autocorrelation) had been checked and we didn't find any problems. Since the effect of related factors might last several days and there is an incubation period of COVID-19 from exposure to infection, we also examined the associations with different lag structures (lag7 and lag14) in the sensitivity analysis. Lag7 refers to a lag of 7 days (median incubation period) and lag14 refers to a lag of 14 days (max incubation period) of COVID-19. GAMs were implemented via the "mgcv" package (version 1.8-28) of R software (version 3.5.2, Vienna, Austria). $P$-value $<0.05$ was considered as statistically significant.

\section{Results}

\section{Comparison of COVID-19 epidemic in BRICS countries}

In BRICS countries, India had the highest totally of confirmed cases with 18.76 million, followed by Brazil (14.45 million), Russia (4.81 million), and South Africa (1.58 million), while China (0.10 million) had the lowest figure as of April 30, 2021 (Table 1). Daily new cases were also highest in India (386 452), followed by Brazil (79 726), Russia (8731), South Africa (1086), and China (33). The total number of administered vaccine doses was highest in China (147.73 million), followed by India (147.73 million), Brazil (35.53 million), and Russia (18.15 million). South Africa had the lowest rate of administered vaccine doses ( 0.18 million) among BRICS countries as of April 30, 2021. Both Brazil and South Africa were in community transmission mode, while there were clusters of cases in the three other countries. There were differing trends in daily new cases among BRICS countries, but all countries had undergone at least two COVID-19 waves from February 2020 to April 2021 (Fig. 1). 
Table 1 Comparison of socio-economic and demographic characteristics, health vulnerability and resources, and policy response to COVID-19 pandemic in BRICS countries

\begin{tabular}{|c|c|c|c|c|c|}
\hline \multirow[t]{2}{*}{ Characteristics } & \multicolumn{5}{|c|}{ BRICS countries } \\
\hline & Brazil & Russia & India & China & South Africa \\
\hline \multicolumn{6}{|l|}{ Socio-economic and demographic characteristics } \\
\hline Total population (millions) & 212.56 & 145.93 & 1380.00 & 1439.32 & 59.31 \\
\hline Population density (people per $\mathrm{km}^{2}$ ) & 25.04 & 8.82 & 450.42 & 147.67 & 46.75 \\
\hline Life expectancy at birth (years) & 75.88 & 72.58 & 69.66 & 76.91 & 64.13 \\
\hline Extreme poverty & 3.40 & 0.10 & 21.20 & 0.70 & 18.90 \\
\hline Human development index & 0.77 & 0.82 & 0.65 & 0.76 & 0.71 \\
\hline GDP per capita (current USD) & 14103.45 & 24765.95 & 6426.67 & 15308.71 & 12294.88 \\
\hline \multicolumn{6}{|l|}{ Health vulnerability } \\
\hline Diabetes prevalence (\%) & 8.11 & 6.18 & 10.39 & 9.74 & 5.52 \\
\hline Cardiovascular death rate (\%) & 177.96 & 431.30 & 282.28 & 261.90 & 200.38 \\
\hline Population aged 60 and above (\%) & 14.05 & 22.41 & 10.12 & 17.35 & 8.54 \\
\hline \multicolumn{6}{|l|}{ Health system resources } \\
\hline Hospital beds per thousand people & 2.20 & 8.05 & 0.53 & 4.34 & 2.32 \\
\hline \multicolumn{6}{|l|}{ Water and sanitation } \\
\hline Access to improved drinking water (\%) & 96.90 & 97.00 & 90.70 & 91.50 & 91.40 \\
\hline Prevalence of open defecation (\%) & 1.13 & 0.00 & 25.73 & 0.00 & 1.45 \\
\hline \multicolumn{6}{|l|}{ Population mobility } \\
\hline Tourist/visitor arrivals (thousands per year) & 6621 & 24551 & 17423 & 62900 & 10472 \\
\hline \multicolumn{6}{|c|}{ Policy response to COVID-19 pandemic as of April 30, 2021} \\
\hline Policy stringency index & 70.83 & 42.13 & 69.91 & 78.24 & 48.15 \\
\hline Total administered vaccine doses (millions) & 35.53 & 18.15 & 147.73 & 211.22 & 0.18 \\
\hline Daily new cases & 79726 & 8731 & 386452 & 33 & 1086 \\
\hline Total confirmed cases (millions) & 14.45 & 4.81 & 18.76 & 0.10 & 1.58 \\
\hline Transmission classification & $\begin{array}{l}\text { Community } \\
\text { transmission }\end{array}$ & Clusters of cases & Clusters of cases & Clusters of cases & Community transmission \\
\hline
\end{tabular}

BRICS Brazil, Russia, India, China, and South Africa, GDP Gross domestic product, COVID-19 Coronavirus disease 2019, USD United States Dollars

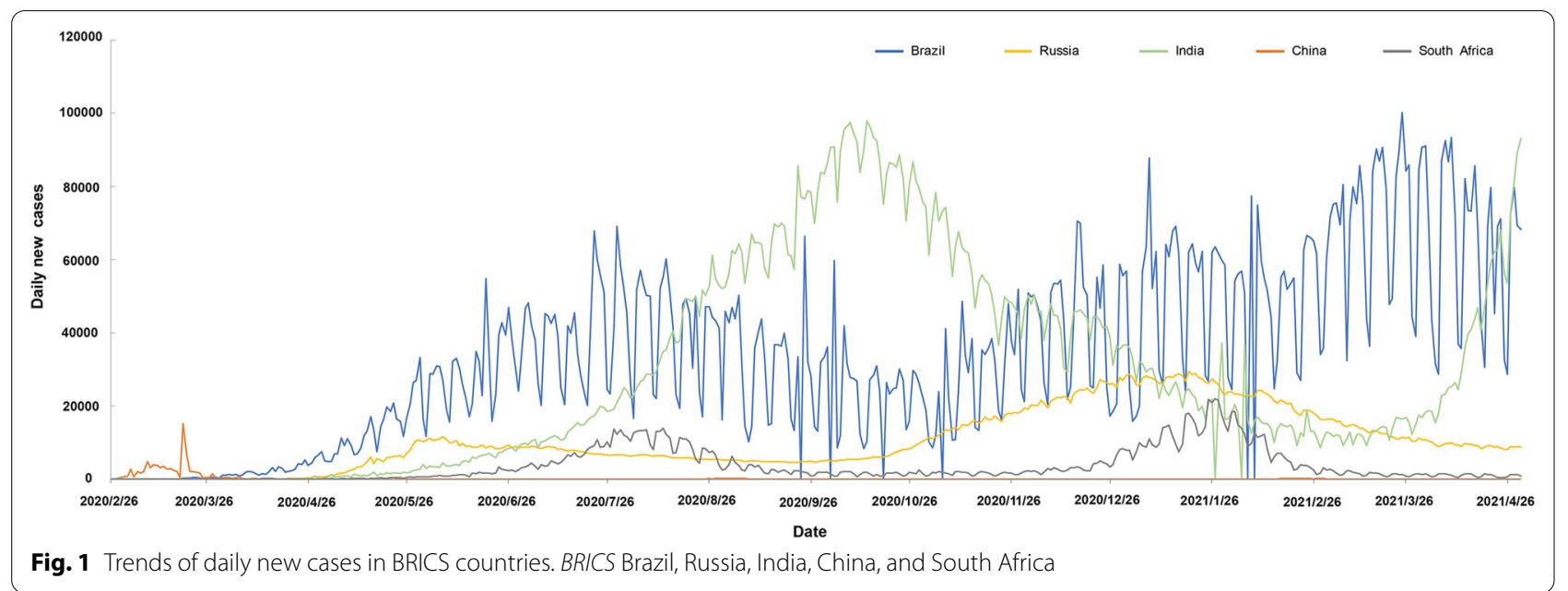

Comparison of socio-economic, demographic characteristics and other indicators in BRICS countries

Table 1 shows the comparison of socio-economic, demographic characteristics and other indicators in BRICS countries. Brazil had the lowest tourist/visitor arrivals. Russia topped the human development index 
and had the least stringent policy. In contrast, China had the largest tourist/visitor arrivals and strictest policy response. On the other hand, India had the highest prevalence rate in open defecation, while its GDP per capita, hospital beds per thousand people and proportion of people with access to improved drinking water were the lowest. South Africa had the smallest population and the lowest proportion of population aged 60 and above.

Table 2 Correlation coefficients among socio-economic and demographic characteristics, health vulnerability and resources, policy response and daily new COVID-19 cases in BRICS countries

\begin{tabular}{lcc}
\hline Variables & \multicolumn{2}{c}{ Daily new cases } \\
\cline { 2 - 3 } & Coefficients & $P$-value \\
\hline Population density (people per km ${ }^{2}$ ) & 0.04 & 0.072 \\
Life expectancy at birth (years) & -0.17 & $<0.001^{* * *}$ \\
Extreme poverty & 0.21 & $<0.001^{* * *}$ \\
Human development index & -0.01 & $<0.001^{* * *}$ \\
GDP per capita (current USD) & -0.09 & $<0.001^{* * *}$ \\
Diabetes prevalence (\%) & 0.13 & $<0.001^{* * *}$ \\
Cardiovascular death rate (\%) & -0.02 & 0.401 \\
Population aged 60 and above (\%) & 0.15 & $<0.001^{* * *}$ \\
Hospital beds per thousand people & -0.17 & $<0.001^{* * *}$ \\
Access to improved drinking water (\%) & -0.30 & $<0.001^{* * *}$ \\
Prevalence of open defecation (\%) & 0.23 & $<0.001^{* * *}$ \\
Tourist/visitor arrivals (thousands per year) & -0.60 & $<0.001^{* * *}$ \\
Policy stringency index & 0.25 & $<0.001^{* * *}$ \\
\hline
\end{tabular}

***P $<0.001$

\section{Correlation coefficients between daily new cases and related indicators}

Daily new COVID-19 cases were correlated with socioeconomic and demographic disadvantages, health and resource vulnerabilities, population mobility, and policy response (Table 2). There was also a positive correlation among daily new COVID-19 cases with population density, extreme poverty, proportion of population aged 60 and above, prevalence of open defecation and policy stringency $(P<0.001)$. On the other hand, life expectancy at birth, human development index, GDP per capita, hospital beds per thousand people, and access to improved drinking water were negatively correlated with daily new COVID-19 cases $(P<0.001)$.

\section{Association of daily new cases with socio-economic and demographic factors and policy response}

In the multivariable GAM model, a 1 unit (people per $\mathrm{km}^{2}$ ) increase in population density was associated with a $5.17 \%$ (lower confidence interval $5.11 \%$, upper confidence interval 5.23\%) growth in daily new COVID-19 cases. In addition, daily new COVID-19 cases (all $P<0.001$ ) were correlated with life expectancy at birth $(-1.61 \%)$, extreme poverty $(8.95 \%)$, human development index $(-0.05 \%)$, GDP per capita $(-0.18 \%)$, diabetes prevalence $(0.66 \%)$, proportion of population aged 60 and above (2.23\%), hospital beds per thousand people $(-0.08 \%)$, proportion of people with access to improved drinking water $(-7.40 \%)$, prevalence of open defecation $(0.69 \%)$, annual tourist/visitor arrivals $(0.003 \%)$ and policy stringency index (1.95\%), after controlling other potential confounders (all $P<0.001$; Table 3).

Table 3 Estimated effects of socio-economic and demographic characteristics, health vulnerability and resources, and policy response on daily new COVID-19 cases in BRICS countries

\begin{tabular}{|c|c|c|c|c|}
\hline \multirow[t]{2}{*}{ Variables } & \multicolumn{4}{|c|}{ Daily new cases } \\
\hline & $\beta$ & $\begin{array}{l}\text { Lower confidence } \\
\text { interval }\end{array}$ & $\begin{array}{l}\text { Upper confidence } \\
\text { interval }\end{array}$ & $P$-value \\
\hline Policy stringency index & $1.95 \%$ & $1.70 \%$ & $2.19 \%$ & $<0.001^{* * *}$ \\
\hline Population density (people per km²) & $5.17 \%$ & $5.11 \%$ & $5.23 \%$ & $<0.001^{* * *}$ \\
\hline Life expectancy at birth (years) & $-1.61 \%$ & $-1.87 \%$ & $-1.36 \%$ & $<0.001^{* * *}$ \\
\hline Extreme poverty & $8.95 \%$ & $7.85 \%$ & $10.04 \%$ & $<0.001^{* * *}$ \\
\hline Human development index & $-0.05 \%$ & $-0.06 \%$ & $-0.04 \%$ & $<0.001^{* * *}$ \\
\hline GDP per capita (current USD) & $-0.18 \%$ & $-0.20 \%$ & $-0.16 \%$ & $<0.001^{* * *}$ \\
\hline Diabetes prevalence (\%) & $0.66 \%$ & $0.58 \%$ & $0.74 \%$ & $<0.001^{* * *}$ \\
\hline Population aged 60 and above (\%) & $2.23 \%$ & $1.96 \%$ & $2.50 \%$ & $<0.001^{* * *}$ \\
\hline Hospital beds per thousand people & $-0.08 \%$ & $-0.09 \%$ & $-0.06 \%$ & $<0.001^{* * *}$ \\
\hline Access to improved drinking water (\%) & $-7.40 \%$ & $-8.35 \%$ & $-6.46 \%$ & $<0.001^{* * *}$ \\
\hline Prevalence of open defecation (\%) & $0.69 \%$ & $0.61 \%$ & $0.77 \%$ & $<0.001^{* * *}$ \\
\hline Tourist/visitor arrivals (thousands per year) & $0.00 \%$ & $0.00 \%$ & $0.00 \%$ & $<0.001^{* * *}$ \\
\hline
\end{tabular}


Different lag structures also indicated similar results on the association of daily new COVID-19 cases with socioeconomic and demographic disadvantages, health and resource vulnerabilities, population mobility, and policy response in the sensitivity analysis (all $P<0.001$ ).

The exposure-response curves showed that the number of daily new COVID-19 cases increased with the rise in policy stringency index. There was a rapid surge in number of daily new COVID-19 cases when the policy stringency index ranged from 0 to 45 . The number of infections climbed slowly when the policy stringency index ranged from 46 to 80 , and decreased when the index was above 80 , after controlling other potential confounders (Fig. 2).

\section{Discussion}

To the best of our knowledge, this is the first study that evaluated the association of daily new COVID-19 cases with socio-economic and demographic factors, health vulnerability, resources, and policy response to the pandemic in BRICS countries. Our findings indicated that socio-economic and demographic factors, health and resource vulnerabilities, population mobility, and policy responses were associated with the COVID-19 pandemic. All results of the GAM model remained stable in the sensitivity analysis. After controlling the effect of other potential confounders, daily new cases had a positive relationship with the policy stringency index when the latter was below 80 . In contrast, there was a negative relationship between daily new COVID-19 cases and the policy stringency index when the index reached above 80 . These results indicated that strong policy response at the national level is crucial to reduce COVID-19 infections and control the pandemic.

Previous findings on the associations among socioeconomic and demographic factors, health vulnerability, resources, and COVID-19 policy response were contentious. Rocha et al. [6] found that the initial spread of COVID-19 infections in the country was most affected by patterns of socio-economic vulnerability rather than population age structure and prevalence of existing chronic diseases. Kayral et al. [20] reported the association between the policy stringency and total cases in E7 countries during the COVID-19 period. In this study, we also found that countries with the most disadvantaged communities, vulnerable health system, and less resources (including health system as well water and sanitation) were associated with more COVID-19 cases in BRICS countries. Our results were consistent with previous findings $[6,20]$. It supported the evidence that socio-economic vulnerability might have impact on the disproportionate burden on states and municipalities.

We found that a 1 unit increase in population density and extreme poverty were associated with a $5.17 \%$ and $8.95 \%$ growth in daily new COVID-19 cases respectively. Furthermore, a 1 percent rise in the proportion of population aged 60 and above and diabetes prevalence were associated with a $2.23 \%$ and $0.66 \%$ increase in daily new COVID-19 cases, respectively. One possible explanation was that the macro socio-economic environment and health system resilience at the national level had a higher impact on the pandemic than individual level, because the impact of individual health vulnerability might be affected by the possibility of exposure to the virus [25].

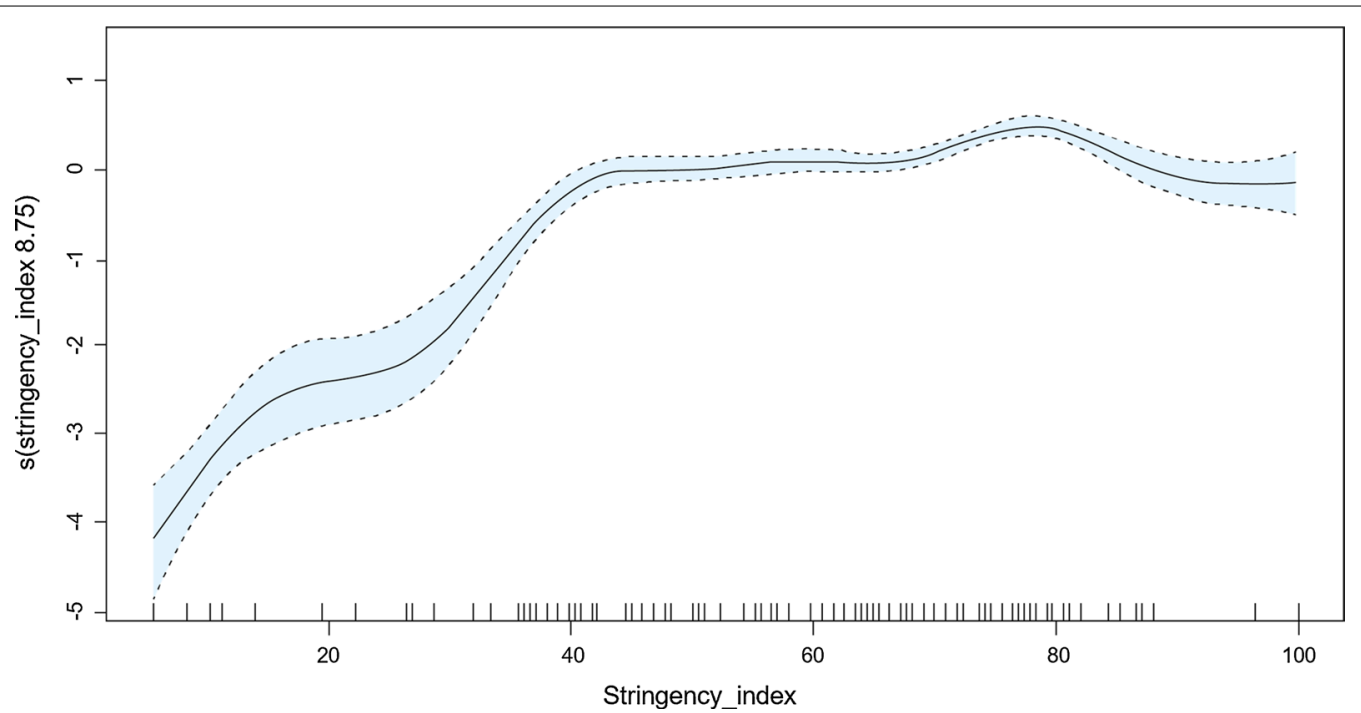

Fig. 2 Exposure-response curves on the effects of policy stringency index in daily new COVID-19 cases. The x-axis represents the policy stringency index. The $y$-axis shows the contribution of the smoother to the fitted values 
Socio-economic and demographic factors, health system vulnerability, as well as lack of water and sanitation resources could reflect the macro environment and system resilience. Mujica et al. found that socio-economic inequalities were associated with health outcomes (mortality) in BRICS countries [21]. Our findings highlighted the importance of reducing socio-economic inequalities and strengthening the resilience of health systems to better respond to public health emergencies in the future.

Brazil, Russia, India, China, and South Africa had nearly $45 \%$ of the world's population [11]. Comparative studies on BRICS countries' health outcomes (such as mortality of chronic diseases) had been conducted but there were no studies focusing on COVID-19. Romaniuk et al. [11] compared the health system outcomes of BRICS countries in 2000-2017 and found that Russia, China, and Brazil had better healthcare performance as opposed to India and South Africa. However, the entire group's overall healthcare performance did not fare well compared to developed countries. They also found that the health system outcomes (such as infant mortality rate and maternal mortality rate) appeared to correlate on a statistically significant scale with GDP per capita [11]. During the COVID-19 pandemic, India, Brazil, and Russia were three of the top five countries with the highest disease burden of COVID-19, along with the United States and France. Moreover, the cumulative total COVID-19 cases in BRICS countries accounted for more than a quarter $(26.3 \%)$ of the global total as of April 30, 2021.

Each BRICS country has its specific characteristics in terms of health performance and policy response to COVID-19 [3-5, 9, 12, 26, 27]. For instance, China had the largest total population with high population mobility and faced significant challenges on COVID-19 prevention and control. China adopted stringent policies and containment strategies, including active case detection and management, lockdown, intercity travel ban, practicing physical distancing and personal protection measures (including hand hygiene, face mask use, etc.). China managed to control its COVID-19 outbreak within three months. While China had the highest absolute number of vaccinations among BRICS countries, it still faced challenges on the relatively low level of vaccination coverage due to its large population. On the other hand, India faced the biggest challenge in coping with COVID-19 among BRICS countries, given its healthcare system vulnerability and high disease burden of COVID-19 as well as low immunization coverage.

There are some limitations in our study. First, COVID19 and related indicators data were based on the national level instead of provincial or city level. We were not able to conduct analysis at provincial or city level. Second, some indicators (such as daily coverage of PCR test on SARS-CoV-2, daily vaccination coverage) were not included in the GAM model due to unavailable data. Third, bilateral correlations were only done in BRICS countries and statistical significance might be underpowered because of limited countries included.

\section{Conclusions}

In BRICS countries, daily new COVID-19 cases were affected by socio-economic and demographic status, health vulnerabilities, resources, and policy response. It is important to reduce socio-economic inequalities and strengthen the resilience of health systems to better respond to public health emergencies. Future studies are needed to explore the way of strengthening public health and health systems resilience in all countries to utilize interventions on curbing the pandemic effectively.

\section{Abbreviations}

COVID-19: Coronavirus disease 2019; SARS-CoV-2: Severe acute respiratory syndrome coronavirus; BRICS: Brazil, Russia, India, China, and South Africa; WHO: World Health Organization; UN: United Nations; GAM: Generalized Additive Model.

\section{Acknowledgements}

Not applicable.

\section{Authors' contributions}

JZ: study design, database establishment, statistical analysis, and paper writing. WY: drew the figures. LZ: manuscript revision. JL: study design, data collection, statistical analysis, and modification of the paper. All authors contributed to manuscript revision, read, and approved the final manuscript.

\section{Funding}

This work was supported by the National Key Research and Development Project of China (2020YFC0846300; 2019YFC1710301), the National Science and Technology Key Projects on Prevention and Treatment of Major Infectious Disease of China (2020ZX10001002) and the National Natural Science Foundation of China (71934002 and 81703240).

\section{Availability of data and materials}

The datasets generated during and/or analyzed during the current study are available in the Our world in Data (https://ourworldindata.org/coronavirussource-data), WHO (https://covid19.who.int/), and UN (http://data.un.org/).

\section{Declarations}

Ethics approval and consent to participate

All analyses in our study were based on public data, thus no ethical approval is required.

\section{Consent for publication}

Not applicable.

\section{Competing interests}

None declared.

\section{Author details}

${ }^{1}$ Department of Economics, University of Birmingham, Birmingham B15 2TT, UK. ${ }^{2}$ Department of Epidemiology and Biostatistics, School of Public Health, Peking University, Haidian District, No. 38, Xueyuan Road, Beijing 100191, China. ${ }^{3}$ Center for Primary Care and Outcomes Research, School of Medicine, Center for Health Policy, Freeman Spogli Institute for International Studies, 
Stanford University, 450 Jane Stanford Way, Stanford, CA 94305-2004, USA. ${ }^{4}$ Institute for Global Health and Development, Peking University, No. 5 Yiheyuan Road, Haidian, Beijing 100871, China. ${ }^{5}$ National Health Commission Key Laboratory of Reproductive Health, Peking University, No. 38, Xueyuan Road, Haidian, Beijing 100191, China.

Received: 13 May 2021 Accepted: 28 June 2021

Published online: 08 July 2021

\section{References}

1. World Health Organization. Health topics/coronavirus. 2021. https://covid 19.who.int/. Accessed 1 May 2021.

2. Kontis V, Bennett JE, Rashid T, Parks RM, Pearson-Stuttard J, Guillot M, et al. Magnitude, demographics, and dynamics of the effect of the first wave of the COVID-19 pandemic on all-cause mortality in 21 industrialized countries. Nat Med. 2020:26:1919-28.

3. Li Z, Chen Q, Feng L, Rodewald L, Xia Y, Yu H, et al. Active case finding with case management: the key to tackling the COVID-19 pandemic. Lancet. 2020;396(10243):63-70

4. Broadbent A, Combrink H, Smart B. COVID-19 in South Africa. Glob Epidemiol. 2020;2: 100034.

5. King EJ, Dudina VI. COVID-19 in Russia: should we expect a novel response to the novel coronavirus? Glob Public Health. 2021. https://doi. org/10.1080/17441692.2021.1900317.

6. Rocha R, Atun R, Massuda A, Rache B, Spinola P, Nunes L, et al. Effect of socioeconomic inequalities and vulnerabilities on health-system preparedness and response to COVID-19 in Brazil: a comprehensive analysis. Lancet Glob Health. 2021. https://doi.org/10.1016/S2214-109X(21) 00081-4.

7. Bauza V, Sclar GD, Bisoyi A, Owens A, Ghugey A, Clasen T. Experience of the COVID-19 pandemic in rural odisha, india: knowledge, preventative actions, and impacts on daily life. Int J Environ Res Public Health. 2021. https://doi.org/10.3390/ijerph18062863.

8. Greer SL, King EJ, Da FE, Peralta-Santos A. The comparative politics of COVID-19: the need to understand government responses. Glob Public Health. 2020;15(9):1413-6.

9. Lancet T. India under COVID-19 lockdown. Lancet. 2020;395(10233):1315.

10. Jefferies S, French N, Gilkison C, Graham G, Hope V, Marshall J, et al. COVID-19 in New Zealand and the impact of the national response: a descriptive epidemiological study. Lancet Public Health. 2020;5(11):e612-23.

11. Romaniuk P, Poznanska A, Brukalo K, Holecki T. Health system outcomes in BRICS countries and their association with the economic context. Front Public Health 2020:8:80.
12. Acharya S, Barber S, Lopez-Acuna D, Menabde N, Migliorini L, Molina J, et al. BRICS and global health. B World Health Organ. 2014;92(6):386.

13. Harmer A, Fleck F. The BRICS countries: a new force in global health? Bull World Health Organ. 2014;92:394-5.

14. Jee M, Or Z. Health outcomes in OECD countries: a framework of health indicators for outcome-oriented policymaking. OECD Labour Market Soc Policy Occas Papers. 1998;36:1-86.

15. Medeiros J, Schwierz C. Efficiency estimates of health care systems. Eur Econ Econ Paper. 2015:549:1-60.

16. Roser M, Ritchie H, Ortiz-Ospina E, Hasell J. Coronavirus pandemic (COVID-19). 2021. https://ourworldindata.org/coronavirus. Accessed 1 May 2021.

17. Dong E, Du H, Gardner L. An interactive web-based dashboard to track COVID-19 in real time. Lancet Infect Dis. 2020;20(5):533-4.

18. Hale T, Angrist N, Goldszmidt R, Kira B, Petherick A, Phillips T, et al. A global panel database of pandemic policies (Oxford COVID-19 Government Response Tracker). Nat Hum Behav. 2021;5(4):529-38.

19. Yan B, Zhang X, Wu L, Zhu H, Chen B. Why do countries respond differently to COVID-19? a comparative study of Sweden, China, France, and Japan. Am Rev Public Admin. 2020;50(6-7):762-9.

20. Kayral IE, Buzrul S. Forecasting of COVID-19 infections in E7 countries and proposing some policies based on the Stringency Index. J Popul Ther Clin Pharmacol. 2020;27(S Pt 1):e76-84.

21. Mujica OJ, Vazquez E, Duarte EC, Cortez-Escalante JJ, Molina J, Barbosa DSJJ. Socioeconomic inequalities and mortality trends in BRICS, 1990-2010. Bull World Health Organ. 2014;92(6):405-12.

22. Talmoudi K, Bellali H, Ben-Alaya N, Saez M, Malouche D, Chahed M. Modeling zoonotic cutaneous leishmaniasis incidence in central Tunisia from 2009-2015: forecasting models using climate variables as predictors. PLoS Neglected Trop Dis. 2017;11(8): e0005844.

23. Wu Y, Jing W, Liu J, Ma Q, Yuan J, Wang Y, et al. Effects of temperature and humidity on the daily new cases and new deaths of COVID-19 in 166 countries. Sci Total Environ. 2020;729:139051.

24. Yuan J, Wu Y, Jing W, Liu J, Du M, Wang Y, et al. Non-linear correlation between daily new cases of COVID-19 and meteorological factors in 127 countries. Environ Res. 2021;193:110521.

25. Legido-Quigley H, Mateos-Garcia JT, Campos VR, Gea-Sanchez M, Muntaner C, McKee M. The resilience of the Spanish health system against the COVID-19 pandemic. Lancet Public Health. 2020;5(5):e251-2.

26. Mckee M, Marten R, Balabanova D, Watt N, Huang Y, Finch AP, et al. BRICS' role in global health and the promotion of universal health coverage: the debate continues. B World Health Organ. 2014:92(6):452-3.

27. Ribeiro H, Lima VM, Waldman EA. In the COVID-19 pandemic in Brazil, do brown lives matter? Lancet Glob Health. 2020;8(8):e976-7.

\footnotetext{
Ready to submit your research? Choose BMC and benefit from:

- fast, convenient online submission

- thorough peer review by experienced researchers in your field

- rapid publication on acceptance

- support for research data, including large and complex data types

- gold Open Access which fosters wider collaboration and increased citations

- maximum visibility for your research: over 100M website views per year
}

At BMC, research is always in progress.

Learn more biomedcentral.com/submissions 